\title{
Sparse Distance Sets in the Triangular Lattice
}

\author{
Tanbir Ahmed \\ Department of Computer Science and Software Engineering \\ Concordia University, Montréal, Canada \\ ta_ahmed@cs.concordia.ca \\ Hunter Snevily \\ Department of Mathematics \\ University of Idaho - Moscow, Idaho, USA \\ snevily@uidaho.edu \\ Submitted: Apr 4, 2013; Accepted: Dec 6, 2013; Published: Dec 17, 2013 \\ Mathematics Subject Classifications: 52B05, 52C10

\begin{abstract}
A planar point-set $X$ in Euclidean plane is called a $k$-distance set if there are exactly $k$ different distances among the points in $X$. The function $g(k)$ denotes the maximum number of points in the Euclidean plane that is a $k$-distance set. In 1996, Erdős and Fishburn conjectured that for $k \geqslant 7$, every $g(k)$-point subset of the plane that determines $k$ different distances is similar to a subset of the triangular lattice. We believe that if $g(k)$ is an increasing function of $k$, then the conjecture is false. We present data supporting our claim and a method of construction that unifies known optimal point configurations for $k \geqslant 3$.
\end{abstract}

\section{Introduction}

For a planar point-set $X$, let $D(X)$ denotes the set of distances between all pairs of points in $X$. A planar point-set $X$ in Euclidean plane is called a $k$-distance set if $|D(X)|=k$. Let $g(k)$ denote the maximum number of points in the Euclidean plane that is a $k$-distance set. Only six exact values are known where $g(1)=3, g(2)=5, g(3)=7, g(4)=9$, and $g(5)=12$ are determined by Erdős and Fishburn [1], and $g(6)=13$ is determined by Wei [2].

Let $R_{n}$ denote the vertices of a regular $n$-gon, and $R_{n}^{+}$denote $R_{n}$ with its center included. Clearly, $g(k) \geqslant 2 k+1$ since $R_{2 k+1}$ is a $k$-distance set. 
The triangular lattice is defined as

$$
L_{\Delta}=\{a(1,0)+b(1 / 2, \sqrt{3} / 2): a, b \in \mathbb{Z}\} .
$$

Given positive integer $a$ and non-negative integers $r_{1}$ and $r_{2}$ with $0 \leqslant r_{2} \leqslant a+r_{1}-1$, let $P_{a, r_{1}, r_{2}}$ denote a $\left(\left(\begin{array}{c}r_{1} \\ 2\end{array}\right)-\left(\begin{array}{c}r_{2} \\ 2\end{array}\right)+a\left(r_{1}+r_{2}\right)+r_{1}-r_{2}+r_{1} r_{2}+a\right)$-point vertically symmetric contiguous and convex subset of $L_{\Delta}$ where rows from top to bottom contain

$$
a, a+1, a+2, \ldots, a+\left(r_{1}-1\right), a+r_{1}, a+r_{1}-1, a+r_{1}-2, \ldots, a+r_{1}-r_{2}
$$

points, respectively.

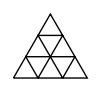

$P_{1,3,0}$

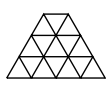

$P_{2,3,0}$

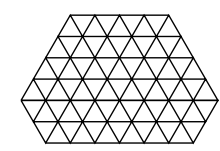

$P_{5,4,2}$

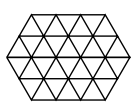

$P_{4,2,2}$

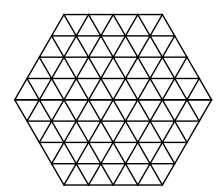

$P_{5,4,4}$

Table 1: Examples of $P_{a, r_{1}, r_{2}}$

Let a saw-blade $B_{s, r, t}$ be an $r$-regular $(r \geqslant 2) s$-sided $(s \in\{3,4,6\})$ polygonal array in $L_{\Delta}$ with $t \leqslant r-1$ triangular blades attached on the first $t+1$ points on each side of the polygonal array. There are $\left(\begin{array}{l}r \\ 2\end{array}\right)+r+3 t, r^{2}+4 t$, and $6\left(\begin{array}{c}r \\ 2\end{array}\right)+6 t+1$ points in $B_{3, r, t}, B_{4, r, t}$, and $B_{6, r, t}$, respectively.

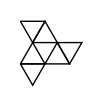

$B_{3,3,1}$

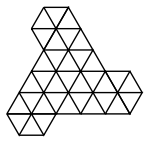

$B_{3,6,2}$

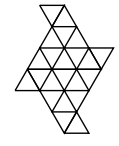

$B_{4,4,1}$

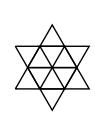

$B_{6,2,1}$

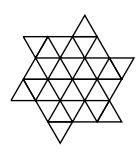

$B_{6,3,1}$

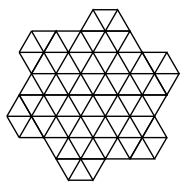

$B_{6,4,2}$

Table 2: Examples of $B_{s, r, t}$

We may generalize the notion of saw-blades and arbitrarily add triangular blades on the sides of $P_{a, r_{1}, r_{2}}$. Typically the tips of consecutive triangular blades are connected by straight lines of unit length. There are $2^{x}$ possible orientations with $x=2 r_{1}+2 r_{2}+(a-$ 1) $+\left(a+r_{1}-r_{2}-1\right)=2 a+3 r_{1}+r_{2}-2$ for $P_{a, r_{1}, r_{2}}$.

Let us call a distance-set sparse if $n / k \geqslant 2.0$, and strictly sparse if $n / k>2.0$. In this paper, we provide examples of sparse distance sets in $L_{\Delta}$ and also present lower bounds of $g(k)$ for $k \leqslant 50$. We also investigate the following conjecture of Erdös and Fishburn.

Conjecture 1 (Erdős and Fishburn [1]). For $k \geqslant 7$, every $g(k)$-point subset of the plane that determines $k$ different distances is similar to a subset of $L_{\Delta}$. 


\section{Computational results}

We can observe the following about computing distance between two points in $L_{\Delta}$.

Observation 1. Let two points in $L_{\Delta}$ be

$$
x \equiv\left(\left(2 a_{1}+b_{1}\right) \frac{1}{2}, b_{1} \frac{\sqrt{3}}{2}\right) \text { and } y \equiv\left(\left(2 a_{2}+b_{2}\right) \frac{1}{2}, b_{2} \frac{\sqrt{3}}{2}\right),
$$

where $a_{1}, b_{1}, a_{2}, b_{2} \in \mathbb{Z}$. Then the square of distance between $x$ and $y$,

$$
d^{2}(x, y)=\left(a_{1}-a_{2}\right)^{2}+\left(a_{1}-a_{2}\right)\left(b_{1}-b_{2}\right)+\left(b_{1}-b_{2}\right)^{2}
$$

involves only integer operations.

Let for $X \subset L_{\Delta}$ on the plane, $D^{2}(X)$ denote $\left\{d^{2}: d \in D(X)\right\}$ and let $D_{i}^{2}(X)$ denote the $i$-th value in the increasing sequence with the values from $D^{2}(X)$.

Let $a_{1}=b_{1}=0$. Then following is a list of the first few values of $D^{2}\left(L_{\Delta}\right)$ (in each case, $d^{2}$ is accompanied by a corresponding pair $\left.\left(a_{2}, b_{2}\right)\right)$ :

\begin{tabular}{rc|ll|lll|ll|ll}
\hline $1:$ & $1(0,1)$ & $11:$ & $25(0,5)$ & $21:$ & $52(2,6)$ & $31:$ & $81(0,9)$ & $41:$ & $112(4,8)$ \\
$2:$ & $3(1,1)$ & $12:$ & $27(3,3)$ & $22:$ & $57(1,7)$ & $32:$ & $84(2,8)$ & $42:$ & $117(3,9)$ \\
$3:$ & $4(0,2)$ & $13:$ & $28(2,4)$ & $23:$ & $61(4,5)$ & $33:$ & $91(1,9)$ & $43:$ & $121(0,11)$ \\
$4:$ & $7(1,2)$ & $14:$ & $31(1,5)$ & $24:$ & $63(3,6)$ & $34:$ & $93(4,7)$ & $44:$ & $124(2,10)$ \\
$5:$ & $9(0,3)$ & $15:$ & $36(0,6)$ & $25:$ & $64(0,8)$ & $35:$ & $97(3,8)$ & $45:$ & $127(6,7)$ \\
$6:$ & $12(2,2)$ & $16:$ & $37(3,4)$ & $26:$ & $67(2,7)$ & $36:$ & $100(0,10)$ & $46:$ & $129(5,8)$ \\
$7:$ & $13(1,3)$ & $17:$ & $39(2,5)$ & $27:$ & $73(1,8)$ & $37:$ & $103(2,9)$ & $47:$ & $133(1,11)$ \\
$8:$ & $16(0,4)$ & $18:$ & $43(1,6)$ & $28:$ & $75(5,5)$ & $38:$ & $108(6,6)$ & $48:$ & $139(3,10)$ \\
$9:$ & $19(2,3)$ & $19:$ & $48(4,4)$ & $29:$ & $76(4,6)$ & $39:$ & $109(5,7)$ & $49:$ & $144(0,12)$ \\
$10:$ & $21(1,4)$ & $20:$ & $49(0,7)$ & $30:$ & $79(3,7)$ & $40:$ & $111(1,10)$ & $50:$ & $147(2,11)$ \\
\hline
\end{tabular}

Let for $X \subset L_{\Delta}, c_{k}^{2}(X)$ denote the number of distinct pairs $(x, y)$ in $X$ such that $d^{2}(x, y)=k$. Let $c^{2}(X)$ denote the sequence $c_{i}^{2}(X)$ for $i=1,2,3, \ldots$

For example, $c^{2}\left(P_{1,2,0}\right)=\langle 9,0,3,3\rangle ; c^{2}\left(P_{2,1,1}\right)=\langle 12,0,6,3\rangle$.

\subsection{Verifying lower bounds of $g(k)$ for $3 \leqslant k \leqslant 13$}

Let $L_{\Delta}^{(n)}$ denote a $\left(n^{2}-n / 2\right)$-point rectangular subset of $n$ consecutive rows in $L_{\Delta}$, with rows having $n, n-1, n, n-1, \ldots$ points, respectively. In this section, we verify the lower bounds of $g(k)$ for $3 \leqslant k \leqslant 13$ by exhaustive search in $L_{\Delta}^{(8)}$.

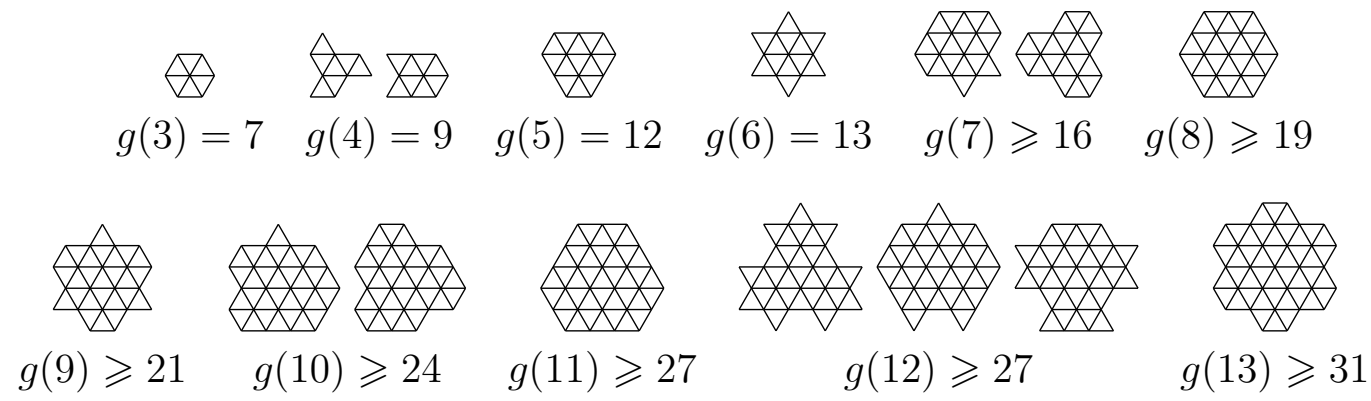


It can be noted that the example of $g(10) \geqslant 25$ in Figure 4 in Erdős and Fishburn [1] is actually an example of $g(11) \geqslant 25$, which can be explained using the example $P_{3,2,2}$ that we have used for $g(8) \geqslant 19$. For $P_{3,2,2}$, we have

$$
D^{2}\left(P_{3,2,2}\right)=\{1,3,4,7,9,12,13,16\} .
$$

Let $Y$ be the set of points when six points corresponding to the blades are added to $P_{3,2,2}$, as in Figure 4 in Erdős and Fishburn [1].

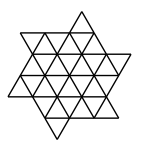

Then we have

$$
D^{2}(Y)=\{1,3,4,7,9,12,13,16,19,21,28\} .
$$

\subsection{Lower bounds of $g(k)$ for $14 \leqslant k \leqslant 50$}

In this section, we provide some new lower bounds of $g(k)$ for $14 \leqslant k \leqslant 50$. The lower bounds for $k=15,23,34$, and 46 were previously known (Erdős and Fishburn [1]). We have considered all possible blade-orientations on the sides of $P_{a, r_{1}, r_{2}}$ for $1 \leqslant a \leqslant 10$, $1 \leqslant r_{1} \leqslant 10$, and $0 \leqslant r_{2} \leqslant a+r_{1}-r_{2}$.

We have also conducted exhaustive searches on the $\left(n^{2}-n / 2\right)$-point rectangular subset of $L_{\Delta}$ for improving lower bounds of smaller values of $k$, say $k=16$. Apparently in $L_{\Delta}$, the bladed $P_{a, r_{1}, r_{2}}$ offer the best lower bounds. In the figures below, every two points at a distance one are joined by a line.
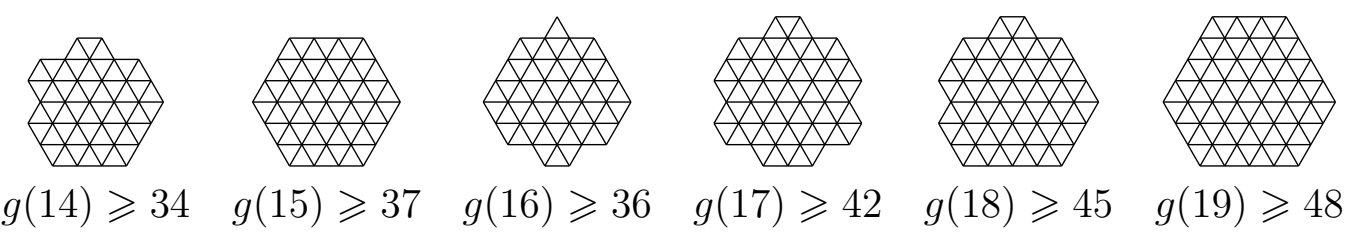

$$
g(14) \geqslant 34
$$
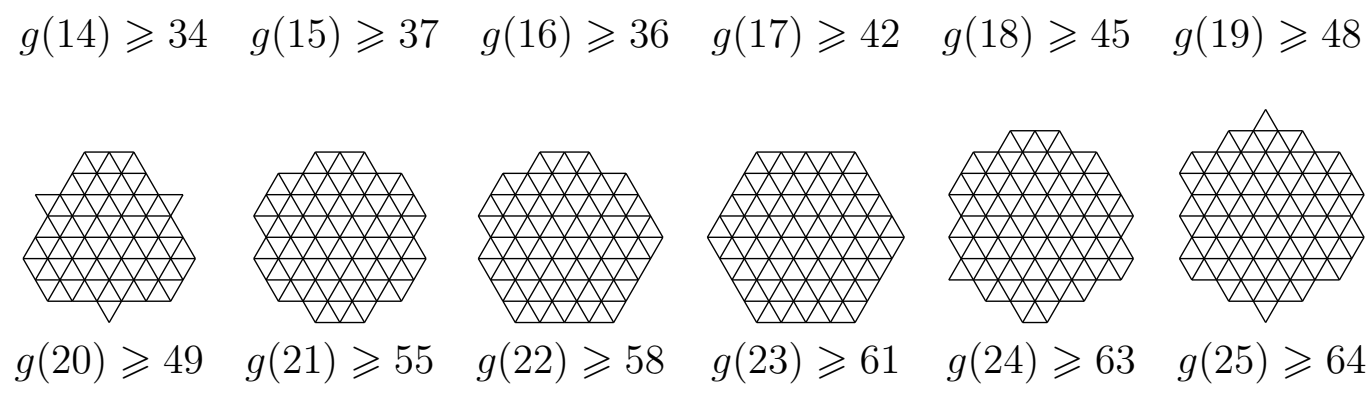

$g(25) \geqslant 64$
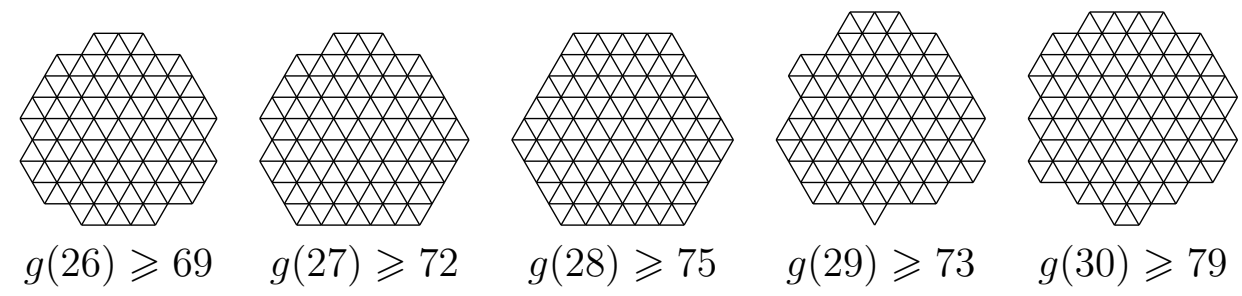

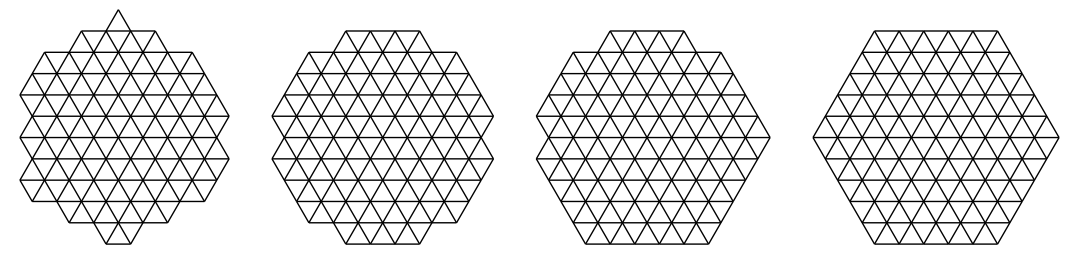

$g(31) \geqslant 80$

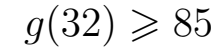

$g(33) \geqslant 88$

$g(34) \geqslant 91$
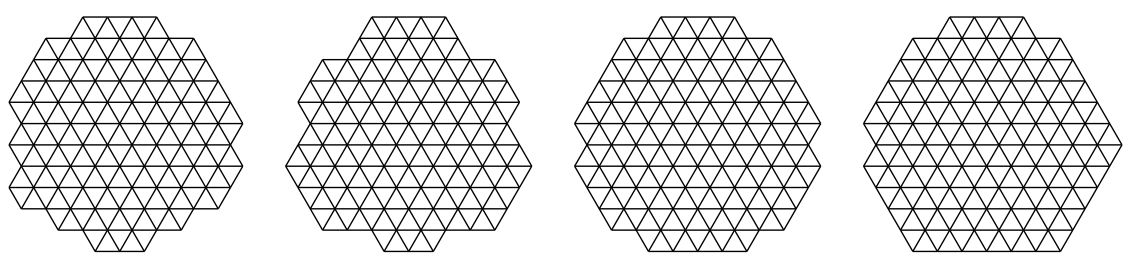

$$
g(35) \geqslant 96
$$
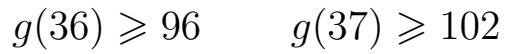

$g(38) \geqslant 105$
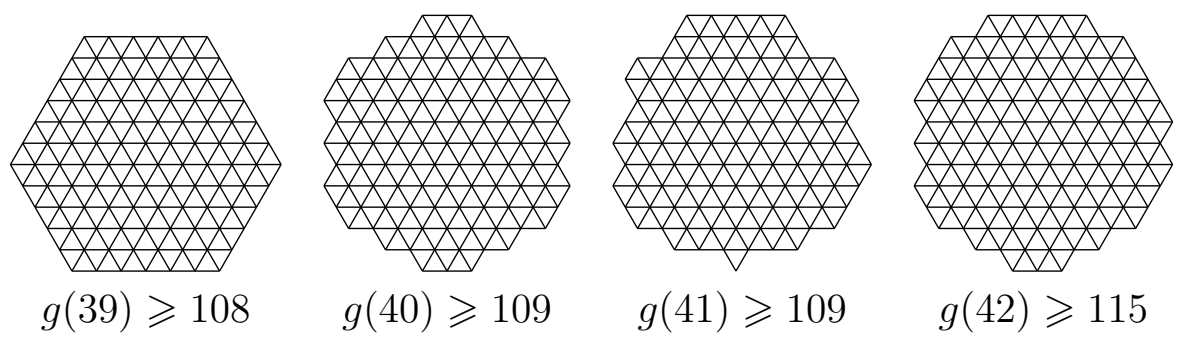

$g(42) \geqslant 115$
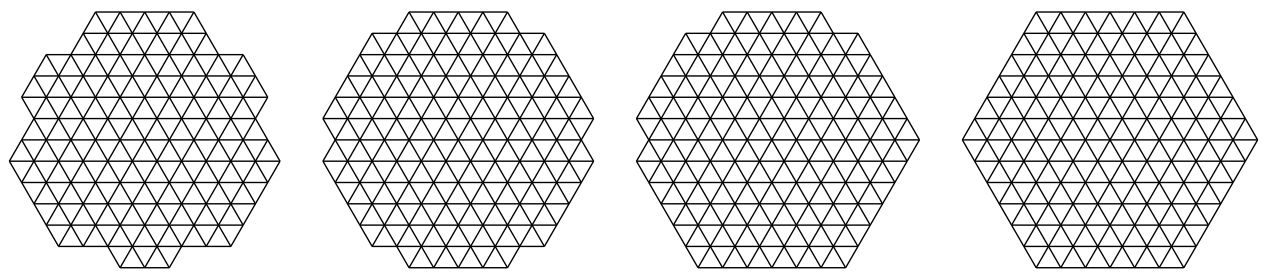

$g(43) \geqslant 115$

$g(44) \geqslant 121$

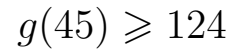

$$
g(46) \geqslant 127
$$

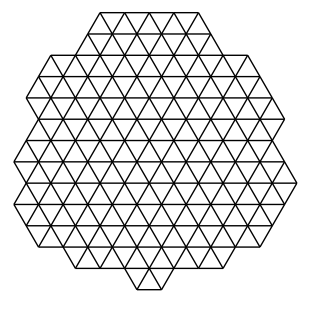

$g(47) \geqslant 129$
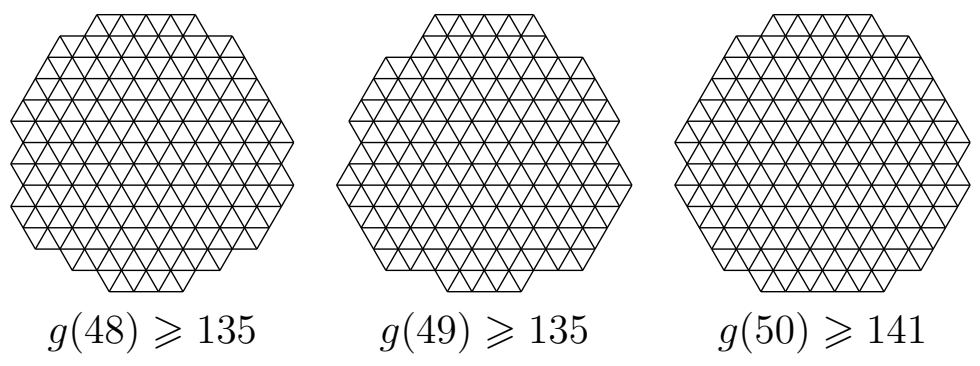

\section{On properties of $P_{a, r_{1}, r_{2}}$ and Bladed $P_{a, r_{1}, r_{2}}$}

Let for $X \subset L_{\Delta}, b(X)$ denote the set of points in $L_{\Delta}$, containing the points on the boundary of $X$. Let $i(X)$ denote the set of points in $L_{\Delta}$, interior to $X$. Clearly, $|X|=$ $b(X)+i(X)$.

Conjecture 2. The number of distinct distances between all pairs of points in $P_{a, r_{1}, r_{2}}$ can be determined by the boundary points of $P_{a, r_{1}, r_{2}}$ alone. (For supporting data, see Appendix E 
Consider the sequences $X$ and $Y$, and let $j$ be the least index such that $X_{j} \neq Y_{j}$. We say $X>Y$ if $X_{j}>Y_{j}$.

Conjecture 3. Given point sets $X$ and $Y$ in $L_{\Delta}$ with $|X|=|Y|$, if $c^{2}(X)>c^{2}(Y)$, then $i(X) \geqslant i(Y)$. (Data in Appendix $\mathrm{D}$ support this conjecture)

Consider the triangular array with points from $P_{r+1, r, 0}$ on the boundary or interior to the triangle, say $T_{r+1, r, 0}$, with vertices

$$
(0,0),(2 r, 0) \text {, and }(3 r / 2,(\sqrt{3} / 2) r) .
$$

Let $p(i, j)$ denote the $j$-th point on the $i$-th row inside $T_{r+1, r, 0}$, considering the base of $T_{r+1, r, 0}$ as the 0 -th row. Let $f(i, j)$ be the square of distance from $(0,0)$ to $p(i, j)$. For example, in $T_{7,6,0}$ in the following figure, $f(2,5)=52$.

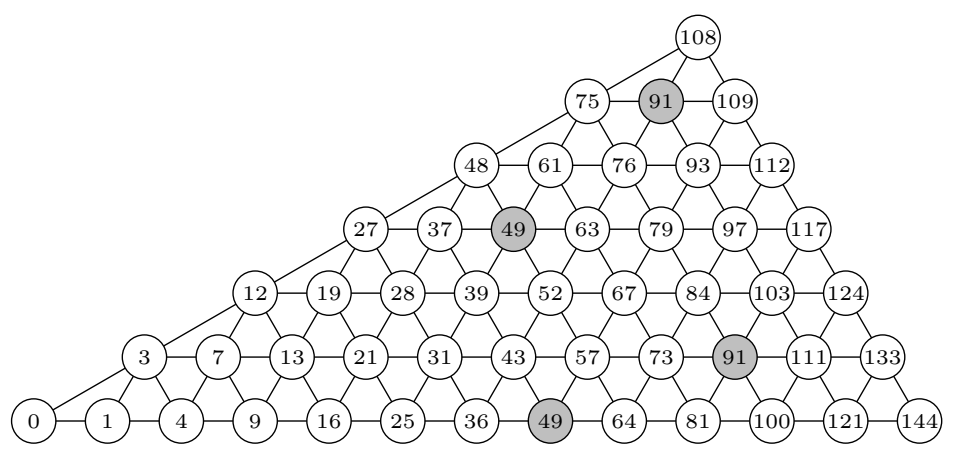

It can be observed that the number of points in $T_{r+1, r, 0}$ is $r^{2}+2 r$. Then for $0 \leqslant i \leqslant r$ and $1 \leqslant j \leqslant(2 r+1)-2 i$,

$$
f(i, j)=\left(\frac{3}{2} i+j-1\right)^{2}+\left(\frac{\sqrt{3}}{2} i\right)^{2} .
$$

Let $r(i, j)$ be the number of times $f(i, j)$ is repeated in $T_{r+1, r, 0}$ above $p(i, j)$. Hence for $r \geqslant 1$,

$$
\left|D\left(P_{r+1, r, r}\right)\right|=\left|D\left(T_{r+1, r, 0}\right)\right|=r^{2}+2 r-\sum_{i=0}^{r} \sum_{j=1}^{2 r+1-2 i}[r(i, j)>0] .
$$

Let $\ell_{\theta}(i, j)$ be the straight line passing through $p(i, j)$ making an angle $\theta \in\{0, \pi / 3,2 \pi / 3\}$ with the positive $x$-axis. Let $d_{\min }(\theta, i, j, k)$ be the smallest square of distance of any point in $T_{r+1, r, 0}$, on the line $\ell_{\theta}(i+k, j-k)$ from $(0,0)$.

\section{Conjecture 4.}

$$
r(i, j)=\sum_{\substack{k \geqslant 1, j+k \leqslant 2 r+1-2 i, d_{\min }(2 \pi / 3, i, j, k) \leqslant f(i, j)}}\left[t(i, j, k) \in \mathbb{Z}^{+}\right],
$$

where $t(i, j, k)$ is a solution to

$$
f(i+k+(t-1), j-k-2(t-1))-f(i, j)=0 .
$$




\section{Questions}

Our data along with Erdős-Fishburn's results and Wei's raise the following questions:

1. Is $g(k)$ an increasing function, i.e., are our configurations for $k=16$ and $k=29$ optimal?

2. Is Conjecture 1 false? This can happen if the answer to Question 1 above is true, but our configuration for $k=16$ is optimal in $L_{\Delta}$. In that case, we need $g(16) \geqslant 37$ in $\mathbb{R}^{2}$.

3. For $k \geqslant 3$, is there always a bladed- $P_{a, r_{1}, r_{2}}$ configuration that is optimal? So far this seems to be the case. Are they always optimal in $L_{\Delta}$ ?

4. Is $D_{k}^{2}\left(L_{\Delta}\right)$ a good approximation of $g(k)$ ? The $D^{2}\left(L_{\Delta}\right)$-table and the lower bounds in Section 2, and the table in Appendix C say this might be the case.

5. Does the equivalent of Conjecture 2 for optimal $g(k)$ configurations hold true? See Appendix $\mathrm{D}$ for supporting data.

\section{Acknowledgements}

The authors would like to thank the Editor and the anonymous referee for their helpful comments and suggestions.

\section{References}

[1] P. Erdős and P. Fishburn, Maximum planar sets that determine $k$ distances, Discrete Math., 160 (1996), 115-125.

[2] X. Wei, A proof of Erdős-Fishburn's conjecture for $g(6)=13$, Elec. J. of Comb., 19(4) (2012), \#P38. 


\section{A 15 -distance sets with 37 -points in $L_{\Delta}^{(8)}$}

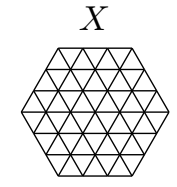

$c^{2}(X)$

$\langle 90,0,72,69,0,0,108,0,48,0,0,39$, $72,0,0,27,0,0,48,0,36,0,0,0,12$, $0,12,18,0,0,12,0,0,0,0,3\rangle$

\section{B 16-distance sets with 36 -points in $L_{\Delta}^{(8)}$}

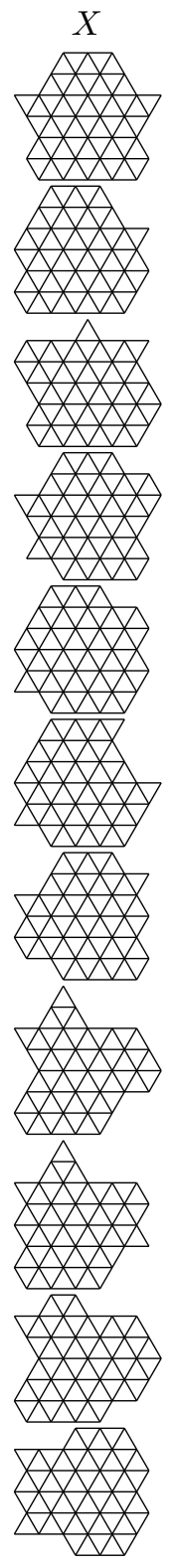

$$
c^{2}(X)
$$

$\langle 85,0,69,64,0,0,104,0,45,0,0,36$, $68,0,0,27,0,0,42,0,36,0,0,0,12$, $0,9,16,0,0,10,0,0,0,0,3,4\rangle$

$\langle 86,0,70,65,0,0,103,0,45,0,0,36$, $67,0,0,27,0,0,42,0,36,0,0,0,12$, $0,9,17,0,0,11,0,0,0,0,2,2\rangle$

$\langle 85,0,69,64,0,0,103,0,45,0,0,36$, $67,0,0,28,0,0,41,0,36,0,0,0,13$, $0,9,16,0,0,10,0,0,0,0,0,5,0,3\rangle$

$\langle 85,0,70,64,0,0,104,0,45,0,0,36$, $68,0,0,27,0,0,42,0,36,0,0,0,12$, $0,9,16,0,0,10,0,0,0,0,2,4\rangle$

$\langle 86,0,71,65,0,0,104,0,45,0,0,36$, $68,0,0,27,0,0,42,0,36,0,0,0,12$, $0,9,16,0,0,10,0,0,0,0,1,2\rangle$

$\langle 85,0,69,65,0,0,102,0,46,0,0,36$, $68,0,0,25,0,0,44,0,34,0,0,0,12$, $0,11,16,0,0,12,0,0,0,0,3,0,0,2\rangle$ $\langle 85,0,71,64,0,0,103,0,45,0,0,36$, $67,0,0,27,0,0,42,0,36,0,0,0,12$, $0,9,17,0,0,11,0,0,0,0,1,4\rangle$

$\langle 84,0,67,62,0,0,101,0,43,0,0,36$, $65,0,0,26,0,0,43,0,36,0,0,0,12$, $0,11,20,0,0,12,0,0,0,0,0,7,0,5\rangle$

$\langle 84,0,69,63,0,0,102,0,45,0,0,36$, $66,0,0,27,0,0,42,0,36,0,0,0,12$, $0,9,18,0,0,12,0,0,0,0,0,6,0,3\rangle$

$\langle 85,0,69,63,0,0,102,0,43,0,0,36$, $66,0,0,27,0,0,42,0,36,0,0,0,13$, $0,11,18,0,0,10,0,0,0,0,0,6,0,3\rangle$ $\langle 85,0,70,64,0,0,104,0,43,0,0,36$, $68,0,0,28,0,0,40,0,36,0,0,0,13$, $0,11,16,0,0,10,0,0,0,0,0,4,0,2\rangle$

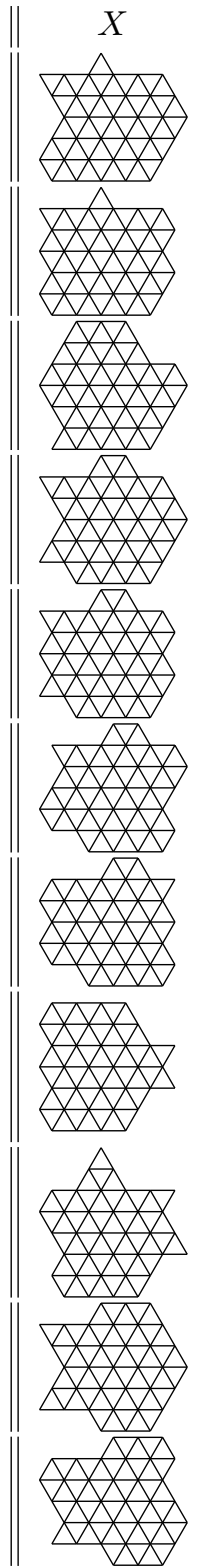

$c^{2}(X)$

$\langle 85,0,68,64,0,0,102,0,45,0,0,36$, $66,0,0,28,0,0,42,0,36,0,0,0,13$, $0,9,16,0,0,10,0,0,0,0,0,6,0,4\rangle$ $\langle 85,0,70,64,0,0,103,0,45,0,0,36$, $67,0,0,28,0,0,41,0,36,0,0,0,13$, $0,9,16,0,0,10,0,0,0,0,0,5,0,2\rangle$

$\langle 86,0,69,65,0,0,103,0,45,0,0,37$, $67,0,0,26,0,0,44,0,34,0,0,0,11$, $0,10,17,0,0,11,0,0,0,0,3,2\rangle$

$\langle 85,0,69,64,0,0,104,0,45,0,0,36$, $68,0,0,28,0,0,40,0,36,0,0,0,13$, $0,9,16,0,0,10,0,0,0,0,0,4,0,3\rangle$ $\langle 85,0,71,64,0,0,104,0,45,0,0,36$, $68,0,0,28,0,0,40,0,36,0,0,0,13$, $0,9,16,0,0,10,0,0,0,0,0,4,0,1\rangle$

$\langle 85,0,70,63,0,0,104,0,45,0,0,36$, $68,0,0,27,0,0,40,0,36,0,0,0,13$, $0,9,18,0,0,10,0,0,0,0,0,4,0,2\rangle$

$\langle 85,0,71,63,0,0,103,0,45,0,0,36$, $67,0,0,27,0,0,41,0,36,0,0,0,13$, $0,9,18,0,0,10,0,0,0,0,0,5,0,1\rangle$

$\langle 85,0,70,64,0,0,102,0,45,0,0,37$, $66,0,0,26,0,0,44,0,34,0,0,0,11$, $0,10,18,0,0,12,0,0,0,0,2,4\rangle$

$\langle 84,0,68,63,0,0,104,0,45,0,0,36$, $68,0,0,27,0,0,40,0,36,0,0,0,12$, $0,9,18,0,0,12,0,0,0,0,0,4,0,4\rangle$

$\langle 85,0,68,64,0,0,104,0,45,0,0,36$, $68,0,0,28,0,0,40,0,36,0,0,0,13$, $0,9,16,0,0,10,0,0,0,0,0,4,0,4\rangle$

$\langle 85,0,69,63,0,0,103,0,43,0,0,36$, $67,0,0,27,0,0,41,0,36,0,0,0,13$, $0,11,18,0,0,10,0,0,0,0,0,5,0,3\rangle$ 


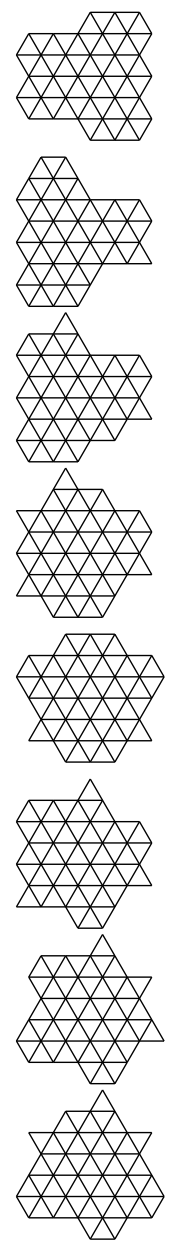

$\langle 85,0,70,62,0,0,102,0,43,0,0,36$, $66,0,0,26,0,0,42,0,36,0,0,0,13$, $0,11,20,0,0,10,0,0,0,0,0,6,0,2\rangle$ $\langle 84,0,68,61,0,0,99,0,41,0,0,36$, $63,0,0,25,0,0,45,0,36,0,0,0,12$, $0,13,22,0,0,12,0,0,0,0,0,9,0,4\rangle$ $\langle 84,0,70,62,0,0,101,0,43,0,0,36$, $65,0,0,26,0,0,43,0,36,0,0,0,12$, $0,11,20,0,0,12,0,0,0,0,0,7,0,2\rangle$ $\langle 84,0,71,64,0,0,103,0,45,0,0,36$, $67,0,0,28,0,0,41,0,36,0,0,0,12$, $0,9,16,0,0,12,0,0,0,0,0,5,0,1\rangle$

$\langle 85,0,71,64,0,0,104,0,45,0,0,36$, $68,0,0,28,0,0,40,0,36,0,0,0,13$, $0,9,16,0,0,10,0,0,0,0,1,4\rangle$

$\langle 84,0,70,63,0,0,104,0,45,0,0,36$, $68,0,0,28,0,0,40,0,36,0,0,0,13$, $0,9,16,0,0,10,0,0,0,0,2,6\rangle$

$\langle 84,0,69,63,0,0,104,0,45,0,0,36$, $68,0,0,28,0,0,40,0,36,0,0,0,13$, $0,9,16,0,0,10,0,0,0,0,3,6\rangle$

$\langle 84,0,70,63,0,0,103,0,45,0,0,36$, $67,0,0,29,0,0,38,0,36,0,0,0,14$, $0,9,17,0,0,11,0,0,0,0,2,6\rangle$

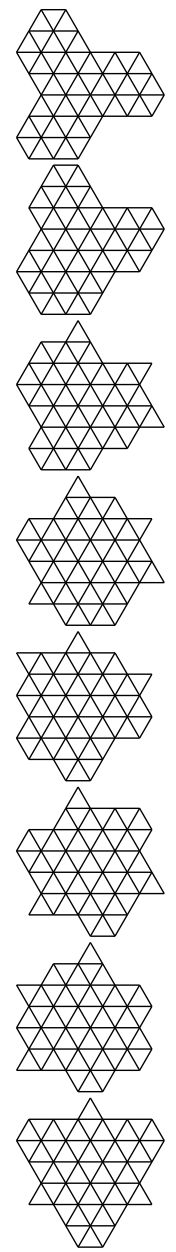

$\langle 84,0,66,60,0,0,96,0,39,0,0,36$, $60,0,0,24,0,0,48,0,36,0,0,0,12$, $0,15,24,0,0,12,0,0,0,0,0,12,0,6\rangle$

$\langle 85,0,68,62,0,0,100,0,41,0,0,36$, $64,0,0,26,0,0,44,0,36,0,0,0,13$, $0,13,20,0,0,10,0,0,0,0,0,8,0,4\rangle$

$\langle 84,0,69,63,0,0,103,0,43,0,0,36$, $67,0,0,27,0,0,41,0,36,0,0,0,12$, $0,11,18,0,0,12,0,0,0,0,0,5,0,3\rangle$

$\langle 84,0,70,64,0,0,104,0,45,0,0,36$, $68,0,0,28,0,0,40,0,36,0,0,0,12$, $0,9,16,0,0,12,0,0,0,0,0,4,0,2\rangle$

$\langle 84,0,71,63,0,0,103,0,45,0,0,36$, $67,0,0,28,0,0,40,0,36,0,0,0,13$, $0,9,17,0,0,11,0,0,0,0,1,6\rangle$

$\langle 84,0,69,63,0,0,105,0,45,0,0,36$, $69,0,0,27,0,0,39,0,36,0,0,0,12$, $0,9,18,0,0,12,0,0,0,0,0,3,0,3\rangle$

$\langle 84,0,71,63,0,0,104,0,45,0,0,36$, $68,0,0,29,0,0,38,0,36,0,0,0,14$, $0,9,16,0,0,10,0,0,0,0,1,6\rangle$

$\langle 84,0,69,63,0,0,102,0,45,0,0,36$, $66,0,0,30,0,0,36,0,36,0,0,0,15$, $0,9,18,0,0,12,0,0,0,0,3,6\rangle$

\section{An approximation for $g(k)$}

We present lower bounds for $g(k)$ with $r$-regular hexagonal arrays $P_{r, r-1, r-1}$ (with $n=$ $6\left(\begin{array}{c}r \\ 2\end{array}\right)+1$ points) for $2 \leqslant r \leqslant 57$, and also compare with the corresponding values of $D_{k}^{2}\left(L_{\Delta}\right)$.

\begin{tabular}{cc|ccc||cc|ccc}
\hline$r$ & $n$ & $k$ & $D_{k}^{2}\left(L_{\Delta}\right)$ & $\left|n-D_{k}^{2}\left(L_{\Delta}\right)\right|$ & $r$ & $n$ & $k$ & $D_{k}^{2}\left(L_{\Delta}\right)$ & $\left|n-D_{k}^{2}\left(L_{\Delta}\right)\right|$ \\
\hline 2 & 7 & 3 & 4 & 3 & 3 & 19 & 8 & 16 & 3 \\
4 & 37 & 15 & 36 & 1 & 5 & 61 & 23 & 61 & 0 \\
6 & 91 & 34 & 93 & 2 & 7 & 127 & 46 & 129 & 2 \\
8 & 169 & 59 & 175 & 6 & 9 & 217 & 74 & 228 & 11 \\
10 & 271 & 90 & 289 & 18 & 11 & 331 & 109 & 351 & 20 \\
12 & 397 & 129 & 432 & 35 & 13 & 469 & 150 & 508 & 39 \\
14 & 547 & 172 & 592 & 45 & 15 & 631 & 196 & 684 & 53 \\
16 & 721 & 222 & 784 & 63 & 17 & 817 & 249 & 889 & 72 \\
18 & 919 & 277 & 999 & 80 & 19 & 1027 & 308 & 1123 & 96 \\
20 & 1141 & 339 & 1252 & 111 & 21 & 1261 & 372 & 1393 & 132 \\
22 & 1387 & 405 & 1531 & 144 & 23 & 1519 & 440 & 1669 & 150
\end{tabular}




\begin{tabular}{ll|ccc||cc|ccc}
24 & 1657 & 477 & 1821 & 164 & 25 & 1801 & 516 & 1987 & 186 \\
26 & 1951 & 557 & 2164 & 213 & 27 & 2107 & 598 & 2331 & 224 \\
28 & 2269 & 639 & 2511 & 242 & 29 & 2437 & 685 & 2707 & 270 \\
30 & 2611 & 731 & 2908 & 297 & 31 & 2791 & 776 & 3099 & 308 \\
32 & 2977 & 822 & 3303 & 326 & 33 & 3169 & 871 & 3508 & 339 \\
34 & 3367 & 925 & 3747 & 380 & 35 & 3571 & 976 & 3969 & 398 \\
36 & 3781 & 1031 & 4225 & 444 & 37 & 3997 & 1088 & 4476 & 479 \\
38 & 4219 & 1139 & 4699 & 480 & 39 & 4447 & 1197 & 4963 & 516 \\
40 & 4681 & 1257 & 5223 & 542 & 41 & 4921 & 1315 & 5488 & 567 \\
42 & 5167 & 1376 & 5776 & 609 & 43 & 5419 & 1440 & 6064 & 645 \\
44 & 5677 & 1504 & 6352 & 675 & 45 & 5941 & 1569 & 6643 & 702 \\
46 & 6211 & 1635 & 6933 & 722 & 47 & 6487 & 1706 & 7267 & 780 \\
48 & 6769 & 1777 & 7588 & 819 & 49 & 7057 & 1844 & 7903 & 846 \\
50 & 7351 & 1914 & 8221 & 870 & 51 & 7651 & 1992 & 8587 & 936 \\
\hline
\end{tabular}

We have corrected some errors in computation in Erdős and Fishburn [1] of the number of distinct distances in $P_{r, r-1, r-1}$ for $14 \leqslant r \leqslant 21$.

\section{Examples of $|D(X)|=|D(B(X))|$}

In this section, for $14 \leqslant k \leqslant 50$ (we have verified for $3 \leqslant k \leqslant 13$ as well), we use the point set $X$ that we used in Section 2.2 to prove $g(k) \geqslant|X|$.
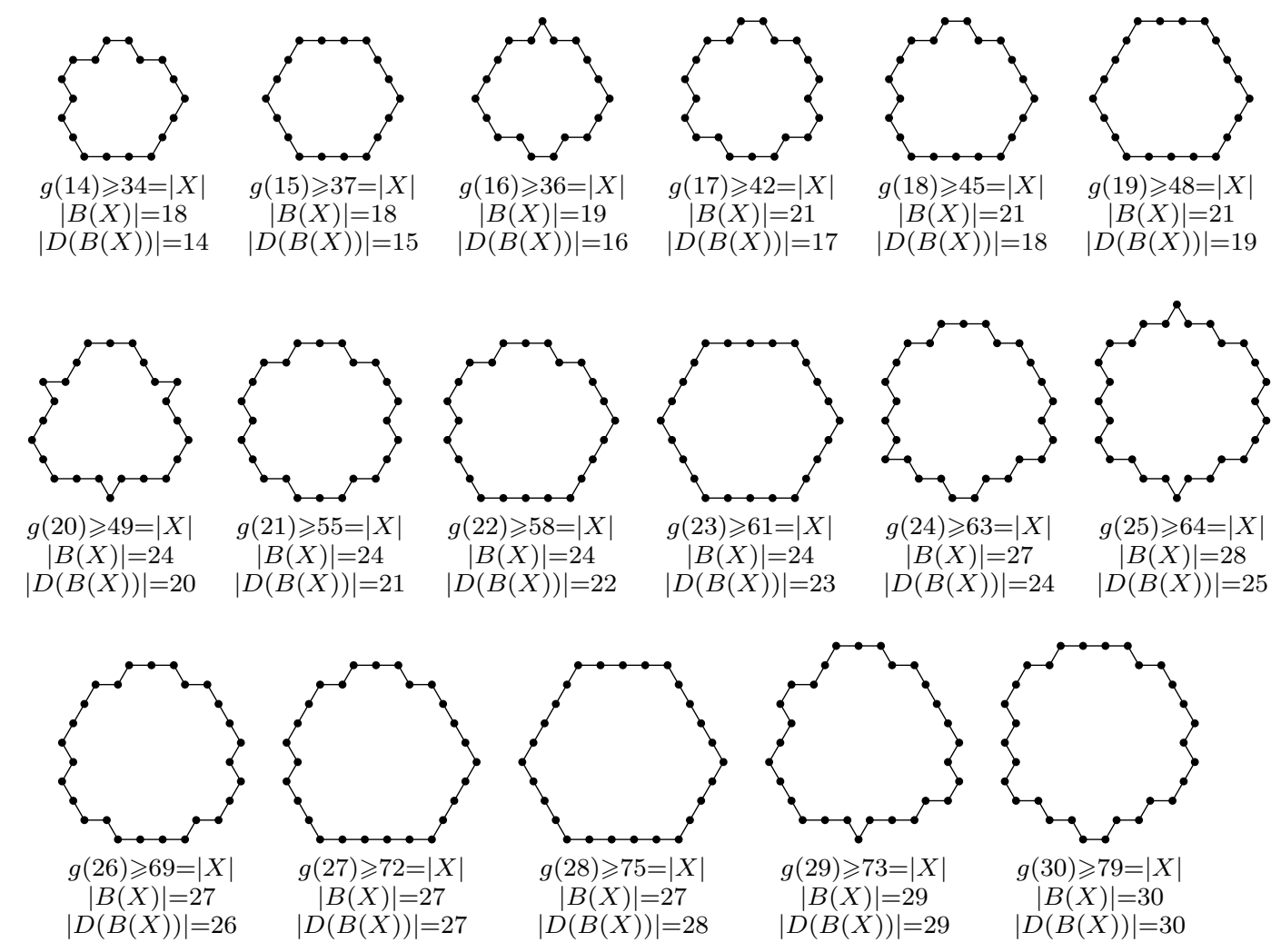

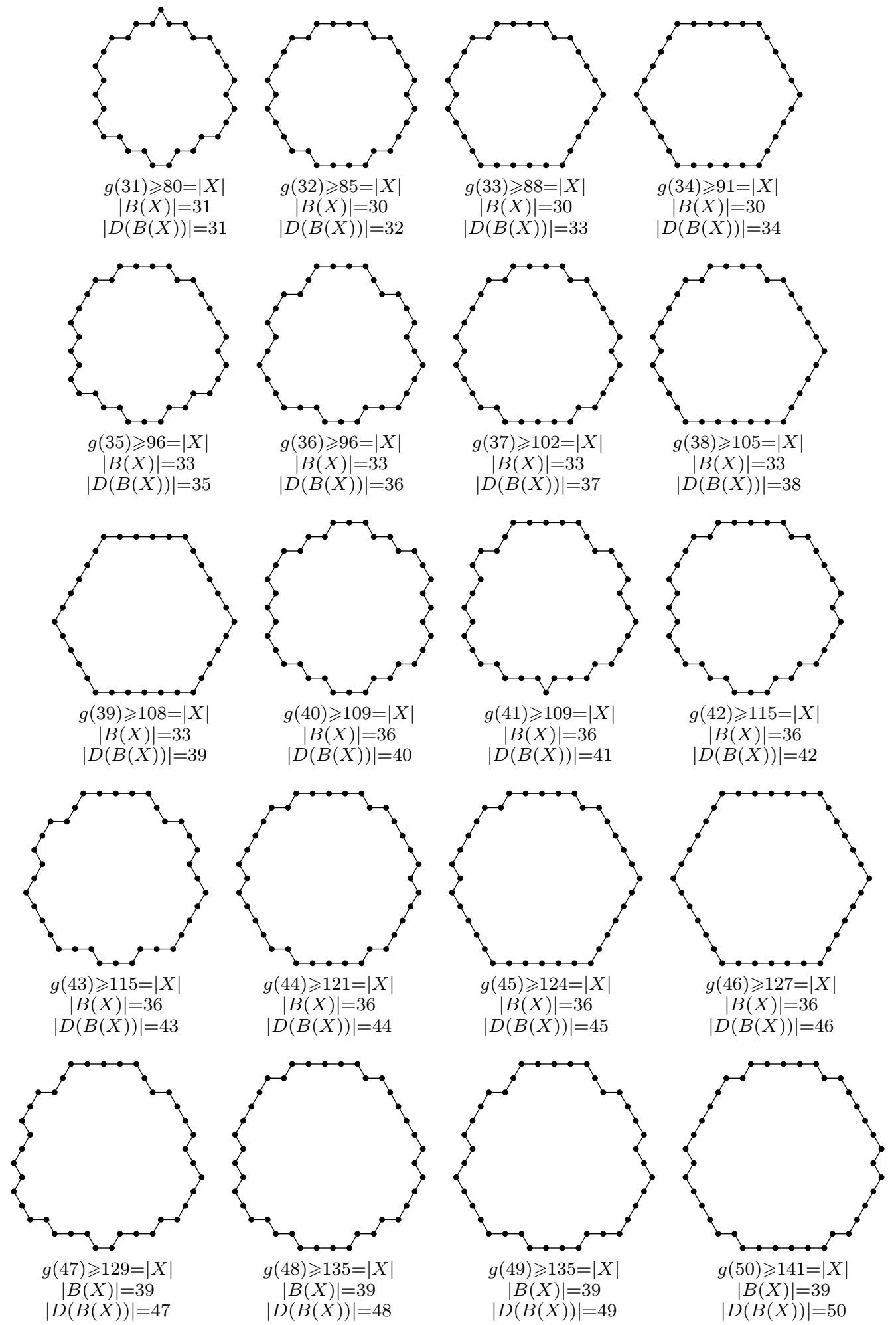

\section{E Data supporting conjecture 2}




\begin{tabular}{|c|c|c|c|c|c|c|c|c|c|}
\hline$X$ & $|X|$ & $|D(X)|$ & $|B(X)|$ & $\mid D(B(X) \mid$ & $X$ & $|X|$ & $|D(X)|$ & $|B(X)|$ & $\mid D(B(X) \mid$ \\
\hline$P_{1,1,0}$ & 3 & 1 & 3 & 1 & $P_{1,1,1}$ & 4 & 2 & 4 & 2 \\
\hline$P_{1,2,0}$ & 6 & 3 & 6 & 3 & $P_{1,2,1}$ & 8 & 4 & 7 & 4 \\
\hline$P_{1,2,2}$ & 9 & 5 & 8 & 5 & $P_{1,3,0}$ & 10 & 5 & 9 & 5 \\
\hline$P_{1,3,1}$ & 13 & 7 & 10 & 7 & $P_{1,3,2}$ & 15 & 8 & 11 & 8 \\
\hline$P_{1,3,3}$ & 16 & 9 & 12 & 9 & $P_{1,4,0}$ & 15 & 8 & 12 & 8 \\
\hline$P_{1,4,1}$ & 19 & 10 & 13 & 10 & $P_{1,4,2}$ & 22 & 12 & 14 & 12 \\
\hline$P_{1,4,3}$ & 24 & 13 & 15 & 13 & $P_{1,4,4}$ & 25 & 14 & 16 & 14 \\
\hline$P_{2,0,1}$ & 3 & 1 & 3 & 1 & $P_{2,1,0}$ & 5 & 3 & 5 & 3 \\
\hline$P_{2,1,1}$ & 7 & 3 & 6 & 3 & $P_{2,1,2}$ & 8 & 4 & 7 & 4 \\
\hline$P_{2,2,0}$ & 9 & 5 & 8 & 5 & $P_{2,2,1}$ & 12 & 5 & 9 & 5 \\
\hline$P_{2,2,2}$ & 14 & 7 & 10 & 7 & $P_{2,2,3}$ & 15 & 8 & 11 & 8 \\
\hline$P_{2,3,0}$ & 14 & 8 & 11 & 8 & $P_{2,3,1}$ & 18 & 8 & 12 & 8 \\
\hline$P_{2,3,2}$ & 21 & 10 & 13 & 10 & $P_{2,3,3}$ & 23 & 12 & 14 & 12 \\
\hline$P_{2,3,4}$ & 24 & 13 & 15 & 13 & $P_{2,4,0}$ & 20 & 11 & 14 & 11 \\
\hline$P_{2,4,1}$ & 25 & 11 & 15 & 11 & $P_{2,4,2}$ & 29 & 14 & 16 & 14 \\
\hline$P_{2,4,3}$ & 32 & 16 & 17 & 16 & $P_{2,4,4}$ & 34 & 18 & 18 & 18 \\
\hline$P_{2,4,5}$ & 35 & 19 & 19 & 19 & $P_{3,0,1}$ & 5 & 3 & 5 & 3 \\
\hline$P_{3,0,2}$ & 6 & 3 & 6 & 3 & $P_{3,1,0}$ & 7 & 5 & 7 & 5 \\
\hline$P_{3,1,1}$ & 10 & 5 & 8 & 5 & $P_{3,1,2}$ & 12 & 5 & 9 & 5 \\
\hline$P_{3,1,3}$ & 13 & 7 & 10 & 7 & $P_{3,2,0}$ & 12 & 8 & 10 & 8 \\
\hline$P_{3,2,1}$ & 16 & 8 & 11 & 8 & $P_{3,2,2}$ & 19 & 8 & 12 & 8 \\
\hline$P_{3,2,3}$ & 21 & 10 & 13 & 10 & $P_{3,2,4}$ & 22 & 12 & 14 & 12 \\
\hline$P_{3,3,0}$ & 18 & 11 & 13 & 11 & $P_{3,3,1}$ & 23 & 11 & 14 & 11 \\
\hline$P_{3,3,2}$ & 27 & 11 & 15 & 11 & $P_{3,3,3}$ & 30 & 14 & 16 & 14 \\
\hline$P_{3,3,4}$ & 32 & 16 & 17 & 16 & $P_{3,3,5}$ & 33 & 18 & 18 & 18 \\
\hline$P_{3,4,0}$ & 25 & 15 & 16 & 15 & $P_{3,4,1}$ & 31 & 15 & 17 & 15 \\
\hline$P_{3,4,2}$ & 36 & 15 & 18 & 15 & $P_{3,4,3}$ & 40 & 18 & 19 & 18 \\
\hline$P_{3,4,4}$ & 43 & 21 & 20 & 21 & $P_{3,4,5}$ & 45 & 23 & 21 & 23 \\
\hline$P_{3,4,6}$ & 46 & 25 & 22 & 25 & $P_{4,0,1}$ & 7 & 5 & 7 & 5 \\
\hline$P_{4,0,2}$ & 9 & 5 & 8 & 5 & $P_{4,0,3}$ & 10 & 5 & 9 & 5 \\
\hline$P_{4,1,0}$ & 9 & 7 & 9 & 7 & $P_{4,1,1}$ & 13 & 8 & 10 & 8 \\
\hline$P_{4,1,2}$ & 16 & 8 & 11 & 8 & $P_{4,1,3}$ & 18 & 8 & 12 & 8 \\
\hline$P_{4,1,4}$ & 19 & 10 & 13 & 10 & $P_{4,2,0}$ & 15 & 11 & 12 & 11 \\
\hline$P_{4,2,1}$ & 20 & 11 & 13 & 11 & $P_{4,2,2}$ & 24 & 11 & 14 & 11 \\
\hline$P_{4,2,3}$ & 27 & 11 & 15 & 11 & $P_{4,2,4}$ & 29 & 14 & 16 & 14 \\
\hline$P_{4,2,5}$ & 30 & 16 & 17 & 16 & $P_{4,3,0}$ & 22 & 15 & 15 & 15 \\
\hline$P_{4,3,1}$ & 28 & 15 & 16 & 15 & $P_{4,3,2}$ & 33 & 15 & 17 & 15 \\
\hline$P_{4,3,3}$ & 37 & 15 & 18 & 15 & $P_{4,3,4}$ & 40 & 18 & 19 & 18 \\
\hline$P_{4,3,5}$ & 42 & 21 & 20 & 21 & $P_{4,3,6}$ & 43 & 23 & 21 & 23 \\
\hline$P_{4,4,0}$ & 30 & 19 & 18 & 19 & $P_{4,4,1}$ & 37 & 19 & 19 & 19 \\
\hline$P_{4,4,2}$ & 43 & 19 & 20 & 19 & $P_{4,4,3}$ & 48 & 19 & 21 & 19 \\
\hline$P_{4,4,4}$ & 52 & 22 & 22 & 22 & $P_{4,4,5}$ & 55 & 25 & 23 & 25 \\
\hline$P_{4,4,6}$ & 57 & 28 & 24 & 28 & $P_{4,4,7}$ & 58 & 30 & 25 & 30 \\
\hline$P_{5,0,1}$ & 9 & 7 & 9 & 7 & $P_{5,0,2}$ & 12 & 8 & 10 & 8 \\
\hline$P_{5,0,3}$ & 14 & 8 & 11 & 8 & $P_{5,0,4}$ & 15 & 8 & 12 & 8 \\
\hline$P_{5,1,0}$ & 11 & 9 & 11 & 9 & $P_{5,1,1}$ & 16 & 11 & 12 & 11 \\
\hline$P_{5,1,2}$ & 20 & 11 & 13 & 11 & $P_{5,1,3}$ & 23 & 11 & 14 & 11 \\
\hline$P_{5,1,4}$ & 25 & 11 & 15 & 11 & $P_{5,1,5}$ & 26 & 14 & 16 & 14 \\
\hline$P_{5,2,0}$ & 18 & 14 & 14 & 14 & $P_{5,2,1}$ & 24 & 15 & 15 & 15 \\
\hline$P_{5,2,2}$ & 29 & 15 & 16 & 15 & $P_{5,2,3}$ & 33 & 15 & 17 & 15 \\
\hline$P_{5,2,4}$ & 36 & 15 & 18 & 15 & $P_{5,2,5}$ & 38 & 18 & 19 & 18 \\
\hline
\end{tabular}




\begin{tabular}{l|l|l||l|l||l|l|l||l|l}
$P_{5,2,6}$ & 39 & 21 & 20 & 21 & $P_{5,3,0}$ & 26 & 19 & 17 & 19 \\
$P_{5,3,1}$ & 33 & 19 & 18 & 19 & $P_{5,3,2}$ & 39 & 19 & 19 & 19 \\
$P_{5,3,3}$ & 44 & 19 & 20 & 19 & $P_{5,3,4}$ & 48 & 19 & 21 & 19 \\
$P_{5,3,5}$ & 51 & 22 & 22 & 22 & $P_{5,3,6}$ & 53 & 25 & 23 & 25 \\
$P_{5,3,7}$ & 54 & 28 & 24 & 28 & $P_{5,4,0}$ & 35 & 23 & 20 & 23 \\
$P_{5,4,1}$ & 43 & 23 & 21 & 23 & $P_{5,4,2}$ & 50 & 23 & 22 & 23 \\
$P_{5,4,3}$ & 56 & 23 & 23 & 23 & $P_{5,4,4}$ & 61 & 23 & 24 & 23 \\
$P_{5,4,5}$ & 65 & 27 & 25 & 27 & $P_{5,4,6}$ & 68 & 31 & 26 & 31 \\
$P_{5,4,7}$ & 70 & 34 & 27 & 34 & $P_{5,4,8}$ & 71 & 37 & 28 & 37 \\
\hline
\end{tabular}

